CAHIERS DE

NARRATOLOGIE

\section{Cahiers de Narratologie}

Analyse et théorie narratives

$13 \mid 2006$

Nouvelles approches de l'intertextualité

\title{
La Voie royale et Voyage au bout de la nuit : deux réécritures françaises de Heart of darkness
}

Isabelle Guillaume

\section{(2) OpenEdition}

Journals

Édition électronique

URL : http://journals.openedition.org/narratologie/331

DOI : 10.4000/narratologie.331

ISSN : 1765-307X

Éditeur

LIRCES

Référence électronique

Isabelle Guillaume, «La Voie royale et Voyage au bout de la nuit: deux réécritures françaises de Heart of darkness », Cahiers de Narratologie [En ligne], 13 | 2006, mis en ligne le 08 septembre 2006, consulté le 30 avril 2019. URL : http://journals.openedition.org/narratologie/331; DOI : 10.4000/narratologie.331

Ce document a été généré automatiquement le 30 avril 2019.

\section{(c) $($ i) $(9)$}

Cahiers de Narratologie - Analyse et théorie narratives est mis à disposition selon les termes de la licence Creative Commons Attribution - Pas d'Utilisation Commerciale - Pas de Modification 4.0 International. 


\title{
La Voie royale et Voyage au bout de la nuit : deux réécritures françaises de Heart of darkness
}

\author{
Isabelle Guillaume
}

1 Il s'agit de lire La Voie royale publiée par André Malraux en 1930 et Voyage au bout de la nuit

${ }^{1}$ que Louis-Ferdinand Céline fait paraître deux ans plus tard comme deux récritures ${ }^{2}$ de Heart of Darkness paru en 1901 et, en traduction, dans des livraisons successives de la N.R.F. , de décembre 1924 à février 1925. Si le témoignage de Clara Malraux permet de savoir que Malraux avait lu le roman de Joseph Conrad avant d'écrire La Voie royale ${ }^{3}$, il est plus difficile d'établir le fait pour Céline. Les deux écrivains français ont en partage d'être très discrets sur le rôle joué par le souvenir de Heart of Darkness dans la genèse de leurs propres œuvres. Pour avoir rendu hommage à un auteur éminemment respecté dans le cercle de la N.R.F., André Malraux n'a pas évoqué précisément sa lecture de Heart of Darkness. Disert quant aux écrivains qui ont pu influencer l'écriture de son premier roman, Céline a tu le nom de Joseph Conrad, citant au gré des ans et des interviews ceux d'Eugène Dabit, de Morand, de Rabelais, de Shakespeare, de Dostoïevski ou de Vallès. Présente au détour de sa correspondance avec l'universitaire américain Milton Hindus, la référence à Joseph Conrad apparaît de manière biaisée sous la forme d'une remarque rapide : « Le nègre du Narcisse a été admirablement traduit en français par Gide - traducteur génial il faut le dire $»^{4}$. Loin de se porter sur le texte, l'intérêt s'attache ici à sa seule traduc tion. Apparemment flatteur, le propos sur André Gide est inséparable des autres commentaires tenus sur l'auteur des Faux-monnayeurs. L'épistolier ne reconnaît la valeur $\mathrm{du}$ traducteur que pour mieux dénigrer la médiocrité du romancier. Si l'on tient La Voie royale pour un texte également connu de Céline ${ }^{5}$, on peut alors constater le même phénomène de glissement dans le système de citation, l'œuvre qui est intervenue dans la genèse de Voyage au bout de la nuit est passée sous silence au profit d'une autre qui lui est davantage étrangère. Le pamphlétaire de Bagatelles pour un massacre admet André Malraux au rang de ses « confrères admirables » mais la référence à $L a$ Voix royale est absente, qui 
cède la place à celle à un autre roman: «Les conquérants de Malraux pour autant que je puisse en juger, voici du chef-d'œuvre ! $»^{6}$.

2 En l'absence de témoignage de la part des auteurs, tandis que la critique a procédé à un repérage d'analogies plutôt rapide ${ }^{7}$, le statut de récriture de $L a$ Voie royale et Voyage au bout de la nuit peut être mis en lumière par une étude comparée du traitement du thème de l'altérité dans les trois fictions. Celles-ci décrivent, en effet, le même parcours théma tique. Les trois récits disent à leur manière que le temps des découvertes est échu et qu'au motif de la découverte de territoires vierges, ce topos de la littérature d'aventure, se substitue celui de l'exploration d'autrui. Dès lors, la traversée de territoires, jungle africaine ou cambodgienne, adopte pour les héros des fictions la forme de la télémachie. A l'horizon du déplacement se dessine une possible communion d'expérience et les trois textes soulignent, selon des modalités qui leur sont propres, l'identité des parcours et des destins. Pourtant, dans le même temps, les narrations se montrent tout aussi attachées à signifier l'opacité d'autrui et les scènes de rencontre qui devraient offrir un point d'orgue aux parcours, s'organisent autour du motif de la lacune. Dès lors, l'enseignement de ces romans qui empruntent volontiers un tour aphoristique est similaire : la fraternité est dénoncée comme une illusion qui dissimule l'expérience authentique de la "séparation ", pour reprendre un des leitmotive orchestrés par La Voie royale.

3 La pénétration, la découverte et la conquête de territoires vierges constituent des topoi de la littérature d'aventure, relations de voyage, récits de découvertes ou romans d'aven tures. Cependant, dans cette fin de $19^{\text {ème }}$ siècle que la fiction de Conrad prend pour cadre, non seulement il ne reste plus de territoires inconnus mais l'inventaire topographique et scientifique a définitivement forclos la terre. Ce type de constat se trouve thématisé dans Heart of Darkness comme dans La Voie royale. Dans le roman de Joseph Conrad, Charles Marlow, héros et narrateur du récit rétrospectif, met en regard ses rêves d'enfant et la réalité qui s'offre à l'adulte qu'il est devenu :

Now when I was a little chap I had a passion for maps [...] At that time there were many blank spaces on the earth [...]. True, by this time it was not a blank space any more. It has got filled since my boyhood with rivers and lakes and names. It had ceased to be a blank space of delightful mystery - a white patch for a boy to dream gloriously over8.

4 La toponymie signe ici la fin du mystère, apanage d'une cartographie muette. De même, pour Claude, avant Kyo à propos duquel le début de La condition humaine rappelle: « depuis plus d'un mois que, de comité en comité, il préparait l'insurrection, il avait cessé de voir les rues : il ne marchait plus dans la boue mais sur un plan $»^{9}$, la voie éponyme se présente d'abord sous les formes de carte et d'inventaire qui nourrissent une obsession. Le motif apparaît de manière récurrente, que la narration épouse le point de vue du héros : «rêver ou lire ? Feuilleter pour la centième fois l'inventaire [...] ? $»^{10}$, tel est le choix qui se propose à Claude, ou qu'elle reste prise en charge par le narrateur anonyme : « Il [Claude] sortit une fois de plus la carte archéologique du Siam et du Cambodge ; il la connaissait mieux que son visage $»^{11}$. Cartes et inventaires sont des motifs absents de Voyage au bout de la nuit qui propose au contraire dans les prémisses de l'épisode africain, l'évocation enthousiaste de «l'Afrique, la vraie, la grande ; celle des insondables forêts, des miasmes délétères, des solitudes inviolées $»^{12}$. La formule de "solitudes inviolées", point d'orgue du mouvement ternaire, semble ici faire écho à celle de Marlow, «that blank space of delightful mystery ». Cependant, la suite du récit de Bardamu confirmera sa valeur de cliché dépourvu de référent véritable. Démystification et subversion du topos du territoire vierge sont des thèmes plus insistants dans le roman de Céline que dans ceux 
de ses prédécesseurs. Tandis qu'au bout de l'Afrique, Bardamu ne trouve rien d'autre que ce qu'il connaissait déjà, l'exotisme apparaît clairement comme un leurre. Déniant l'étrangeté des lieux, le narrateur ne cesse de ramener l'autre au connu, évoquant en ces termes la capitale coloniale : « seule cette crudité de verdure inouïe empêchait l'endroit de ressembler tout à fait à la Garenne-Bezons " ${ }^{13}$, « la végétation bouffie des jardins tenait à grand-peine, agressive, farouche, entre les palissades, éclatantes frondaisons formant laitues en délire ${ }^{14}$, « la place Faidherbe possédait sa forte ambiance, son décor poussé, sa surabondance végétale et verbale de sous-préfecture du midi $\aleph^{15}$, soulignant à propos de la jungle africaine dont il refuse de faire le lieu de l'altérité radicale : " dans leur creux [des arbres abattus] un métro entier aurait manœuvré à son aise $»^{16}$ ou encore "j'étais servi moi qui n'aimais pas la campagne $»^{17}$.

Dès lors, dans les trois romans, le thème de l'exploration d'autrui se substitue à celui de l'exploration de terres inconnues. A cet égard, on peut signaler le caractère totalement amphibologique du titre du roman de Joseph Conrad. À comparer celui-ci et celui de Voyage au bout de la nuit, il apparaît que les termes de « darkness » et de " nuit » jouent sur le même symbolisme polysémique ${ }^{18}$. Cependant «Heart of Darkness» est encore plus complexe dans la mesure où l'absence de préposition et l'emploi du génitif redoublent l'ambiguïté sémantique d'une amphibologie grammaticale. Charles Marlow se rend « au plus profond des ténèbres » à seule fin de rencontrer Kurtz, ce "cœur ténébreux ». L'analogie figure aussi dans le premier chapitre de La Voie royale, sous la forme d'une métaphore spatiale qui rend compte de l'évolution des rapports des deux passagers: "Comme si tous deux se fussent exprimés par paraboles, ils [Claude et Perken] s'approchaient de plus en plus l'un de l'autre cachés par les souvenirs $»^{19}$. Plus discrètement, dans Voyage au bout de la nuit, l'expression, "ce ténébreux ${ }^{20}$, utilisée comme substitut lexical de Robinson au moment de la découverte de celui-ci au fond de la jungle africaine, file la métaphore du titre pour suggérer que la rencontre de l'employé de la factory constitue le véritable accès au « bout de la nuit ».

6 Au cours du voyage au cœur du Congo belge, l'indifférence première de Marlow à l'égard de l'individu dont il doit rejoindre le comptoir cède la place à une obsession soulignée par un adverbe en position détachée dans : « for me it [the steamboat] crawled towards Kurtz - exclusively $»^{21}$. La Voie royale propose par rapport à son hypotexte un dédoublement supplémentaire puisqu'il met en récit un prisonnier, Grabot, et deux héros qui partent à la recherche de celui-ci, Claude et Perken. Dans une fiction qui est celle de toutes les répétitions, l'objet de la quête semble d'abord se dédoubler, les statues khmères pour Claude, Grabot pour Perken. Pourtant, la troisième partie fait état de la «hantise » qui s'empare de Claude et le terme fait écho à celui d'« obsession » utilisé dans la première partie à propos la Voie royale: «Les photos rapportées de Bangkok par Perken [sur lesquelles figure Grabot] vivaient en Claude avec l'autorité de la hantise »². Après Grabot, le personnage de Robinson campe un nouveau Kurtz, dans Voyage au bout de la nuit. Être de fuite et figure mystérieuse, il disparait du comptoir africain au moment où Bardamu retrouve son identité à la faveur d'une étrange réminiscence. Celle-ci détermine le début de l'obsession que le héros de Céline a en partage avec ceux des deux autres romanciers.

Dans les trois récits, le déplacement géographique, remontée de fleuves africains pour Marlow et Bardamu, traversée de la jungle cambodgienne pour Claude et Perken, se fait pour retrouver un autre. C'est ainsi que le héros de Conrad met ses pas dans les empreintes laissées par le chasseur d'ivoire. Empruntant la direction du cœur des ténèbres, son itinéraire personnel adopte la forme de la répétition, du pèlerinage ou de la 
télémachie, pour reprendre un terme de Michel Serres qui lançait cette interrogation rhétorique à propos de l'œuvre de Jules Verne: "Après Ulysse ou Gilgamesh, après Saknussemm ou Livingstone, peut-on écrire plus qu'une Télémachie ? ${ }^{23}$. La formule rend aussi bien compte de La Voie royale au début duquel la parole revient au personnage de Perken qui livre la raison qui le détermine à accompagner Claude dans sa recherche des temples Khmers : « je vais chercher - rechercher - un homme pour qui j'avais une grande sympathie et une grande méfiance ${ }^{24}$. De même, à reprendre la chute du chapitre 13 de Voyage au bout de la nuit, le parcours de Robinson sert à baliser celui de Bardamu : "Je décidai [...] de prendre la forêt devant moi dans la direction qu'avait prise déjà ce Robinson de tous les malheurs $»^{25}$. Bardamu calque ainsi sa conduite sur celui qui, avant lui, a déserté la factory. Il semble alors que le terme de la quête des héros des romans soit voué à prendre la forme d'une communion d'expériences, l'épisode clé des récits, à se confondre avec une scène de rencontre tandis que les héros des trois romans pourraient reprendre à leur compte la formule « je est un autre».

itinéraires de Kurtz, de Grabot ou de Robinson seraient autant d'annonces du destin de ceux qui font figure de nouveaux Télémaque. Le récit de Marlow, dans Heart of Darkness ne commence pas ex abrupto mais il est précédé d'une réflexion historique prenant appui sur deux paraboles. L'exemple le plus développé met en scène un jeune Romain au contact de "the wilderness ", appelée aussi "the savagery " : « it has a fascination, too, that goes to work upon him $»^{26}$. Ces origines de la narration de la rencontre de Kurtz confèrent au parcours de celui-ci le caractère d'un paradigme et suggèrent que tout Européen peut être amené à mettre ses pas dans ses empreintes. C'est ce que qu'invitent également à penser les propos éminemment pessimistes du médecin de la "porte des Ténèbres ", le siège de la société bruxelloise où Marlow signe son contrat de capitaine de vapeur : «the mental changes on the spot $»^{27}$, annonce le praticien avec un enthousiasme certain. Énonçant la loi de l'Afrique, le discours médical fonctionne aussi comme une prolepse à laquelle viendra répondre une analepse au moment où Marlow se rendra compte des effets destructeurs du contact avec cette terre de ténèbres qu'est le Congo belge. C'est ainsi que la crise de folie meurtrière qui s'empare du colon blanc en compagnie duquel Marlow se voit forcé de traverser les terres pour rejoindre le poste central donne lieu à cette mise en perspective : «I remembered the old doctor, - 'it would be interesting for science to watch the mental changes of individuals, on the spot $»^{28}$.

Dans $L a$ Voie royale, avant Grabot, Perken fait figure de prédécesseur pour Claude, à propos duquel le narrateur évoque "la curiosité angoissée qui le poussait vers lui [Perken] comme s'il eût prophétiquement vu son propre destin $»^{29}$. A cet égard, Gaëtan Picon écrit dans la monographie qu'il consacre à l'auteur des Conquérants : «On a souvent remarqué que les personnages se différencient dans la mesure où ils s'opposent et incarnent des vérités inconciliables. On a moins remarqué qu'ils appartiennent à des étapes différentes de la formation humaine, et qu'il y a entre eux la distance de celui qui a subi l'épreuve à celui qui ne l'a pas encore subie. La distance entre Garine et le narrateur, entre Perken et Claude, n'est nullement une opposition [...] mais une hiérarchie : distance de l'adolescent à l'homme mûr et, sinon du disciple au maitre, du moins fort au plus fort " ${ }^{30}$. Que Robinson fait aussi figure de mentor pour celui qui le suit, c'est ce que souligne le constat de Bardamu : « Décidément d'avoir suivi dans la nuit Robinson jusque là où nous en étions, j'avais quand même appris des choses " $^{31}$.

10 Si l'âge désigne à la fonction d'initiateur le personnage de Perken, il semble pourtant que la relation entre celui-ci et Claude soit moins cette relation de «l'adolescent à l'homme 
mûr et, sinon du disciple au maître, du moins fort au plus fort » évoquée par Gaëtan Picon qu'un rapport en miroir entre deux héros qui ont en partage la même obsession de la mort, angoisse et refus mêlés. Cette similitude est mise en valeur dans un roman qui compte de nombreux passages au style direct ; l'identité des locuteurs n'est souvent pas précisée et ce silence du narrateur a pour effet de souligner la familiarité des conceptions des personnages. De plus, la pauvreté lexicale, la manière dont les paragraphes, juxtaposés plus que liés, font se succéder le récit et les dialogues et des sujets sans lien apparent, contribuent à donner l'impression qu'un seul discours se trame dans l'espace du roman.

11 L'autre apparaît alors comme le révélateur du refoulé, comme celui qui permet l'accès à une vérité intime. Pour Marlow, Kurtz incarne déjà la tentation de succomber au vertige d'une sauvagerie dont il sent la menace latente, à la vue de danses indigènes sur le rivage par exemple. Si le narrateur privilégié de Joseph Conrad se trouve pris d'une sorte de vertige devant l'histoire d'autrui qu'il a commencé à raconter, histoire du chasseur d'ivoire ou de Jim dans un autre roman de la trilogie de $\mathrm{Marlow}^{32}$, c'est que cet autre renvoie à lui-même et à ce qu'il refuse de reconnaître en lui. C'est cette identité que soulignent encore dans $\mathrm{L} a$ Voie royale tous les échos réveillés chez Claude par la parole de Perken, que celle-ci le ramène à son histoire familiale ou à son refus du vieillissement, cette déchéance. A cet égard, on peut relever, surtout au début du roman, une belle fréquence des formules : «Claude connaissait $»^{33}$, «y appela un souvenir $~_{34}$, «Claude se souvint $»^{35}$, « que Perken parlât de lui-même et il faisait surgir en Claude ${ }^{36}$, « et Claude, en face de Perken, retrouvait $\aleph^{37}$, "Claude [...] revit sa mère ${ }^{38}$. De manière plus paradoxale, le désir qui s'empare de Claude à la vue de l'humiliation de Grabot de «supprimer ce visage, cette haine, cette présence, $-[. .$.$] chasser cette preuve de sa$ condition d'homme ${ }^{39}$ procède aussi de la prise de conscience d'une identité.

De même, dans Voyage au bout de la nuit, Bardamu ne trouve au terme de son parcours à travers la forêt africaine que son "semblable, son frère", ce Robinson qui, pour emprunter son nom au héros de Defoe, fait figure d'anti-colon et laisse sa factory partir à vau l'eau. Parmi les marchandises dont il se trouve légataire figure « une carte postale en couleurs : 'la place de Clichy' $»^{40}$ qui renvoie symboliquement au cadre de l'incipit, au lieu de l'origine du parcours où «ça a commencé comme ça »" . Une fois rejoints Robinson et sa «demeure presque théorique, effilochée de partout $»^{42}$, le déplacement dans la jungle africaine se transforme en anamnèse, voyage intérieur dans l'espace de la mémoire : « Ce nom de Robinson finit cependant à force de m'entêter par me révéler un corps, une allure, une voix même que j'avais connus... [...] Tout est revenu. Des années venaient de passer d'un seul coup $»^{43}$. La rencontre avec l'autre prend le tour paradoxal d'une confrontation avec soi sous la forme d'une remontée du passé : rappel des antiennes maternelles, dictons tenant lieu de principes moraux, souvenir de l'incendie de la Société des Téléphones qui l'a marqué dans son enfance, « l'année d'avant l'exposition, [...] quand j'étais encore bien petit $~_{44}$. Loin de permettre l'appréhension d'une altérité, le périple africain suscite la résurgence du roman familial. La suite du roman confirmera le sens de cet épisode, Robinson est bien le miroir que Bardamu promène sur sa route. Les expériences des deux personnages se font écho et se redoublent. On a pu dire qu'à partir d'un certain point Robinson prend le relais de Bardamu, continuant à être traqué lorsque celui-ci cesse de l'être, refusant, à la fin du roman, la sécurité avec Madelon, comme Bardamu l'a refusée avec Molly. Si La Voie royale et Voyage au bout de la nuit font appel, à la suite d'Heart of Darkness, à la structure d'une télémachie qui trouverait sa consécration 
dans une communauté d'expérience, si les trois récits invitent à repérer l'identité des parcours et des destins, ces suggestions constituent seulement un des aspects de romans essentiellement complexes et tout aussi attachés à dire l'opacité de l'autre.

Le thème de l'opacité d'autrui peut prendre la forme du motif, récurrent d'un roman à l'autre, des légendes qui se trament autour des figures qui, nourrissant la fascination, font figure d'objet de la quête. La recherche s'accomplit dans l'espace pour Marlow et Bardamu suivant Kurtz ou Robinson, pour Claude et Perken recherchant Grabot, Claude se trouve d'emblée en présence de Perken, reste pour tous les personnages à dépasser le bruit de la rumeur qui voile la vérité de l'individu. Orchestré dans les trois romans, le motif apparaît à trois reprises au début de La Voie royale à propos de Perken : « la légende que faisait rôder autour de cette silhouette confuse les passagers avides de potins et de manilles, la trame des bavardages, de romans et de rêveries qui accompagne les blancs qui ont été mêlés à la vie des états indépendants d'Asie " $^{45}$, "La légende de Perken, maintenant rôdait dans le bateau, passait de chaise longue à chaise longue comme l'angoisse ou l'attente de l'arrivée, comme l'ennui malveillant des traversées. Toujours informe $~_{46}$, «On parlait des missions que le Siam lui avaient confiées auprès des tribus insoumises, de son organisation du pays sham et des marches laotiennes, de ses rapports singuliers avec le gouvernement de Bangkok... $»^{47}$.

Le motif est bien emprunté au roman de Conrad dans lequel les étapes de Marlow vers Kurtz s'assortissent d'autant de propos tenus sur le chasseur d'ivoire ${ }^{48}$. Tout aussi redevable, à cet égard, à Heart of Darkness, le récit de Voyage au bout de la nuit propose, au sujet de l'employé que Bardamu doit remplacer, tout un réseau d'annonces. Celles-ci prennent d'abord la forme d'invectives lancées par le peu amène directeur de la Compagnie Pordurière : «cet autre farceur $»^{49}$, « un beau salaud», «ce fumier-là ! $»^{50}$, "l'autre numéro", «la carne ", «le beau fumier " Gono, cet avant poste du progrès, pour reprendre le titre ironique d'une nouvelle de Conrad $^{52}$, Bardamu tente vainement de tirer partie des discussions filandreuses des colons à l'heure de l'apéritif :

Mais le temps passait et ni les uns ni les autres de ces compagnons ne pouvaient me dire à quel genre d'original exactement appartenait l'individu que j'allais remplacer à Bikomimbo.

«C'est un drôle de type ! » m'avertissaient-ils, et c'était tout ${ }^{53}$.

J'effectuai une dernière fois le tour de mes petits camarades de la Pordurière pour tenter de me renseigner sur le compte de cet employé infidèle, celui que je devais aller coûte que coûte, selon les ordres, remplacer dans sa forêt. Vains bavardages. Le café Faidherbe, au bout de l'avenue Fachoda bruissait vers l'heure du crépuscule de cent médisances, ragots et calomnies, ne m'apportait rien non plus de substantiel. Des impressions seulement ${ }^{54}$.

Dans les trois romans, et plus clairement chez Conrad et Céline ${ }^{55}$, le traitement du motif de la rumeur participe aussi d'une satire sociale, qui dénonce la nullité intellectuelle des colons. Cette inscription de propos tenus par la voix collective et, partant, sujets à caution pourrait valoir comme étape dans le trajet des héros vers la vérité des êtres. Cependant, la scène de rencontre se révèle décevante, qui montre que la connaissance d'autrui n'est qu'un leurre. A cet égard, Heart of Darkness, La Voie royale et l'épisode africain dans Voyage au bout de la nuit s'organisent autour d'une lacune centrale.

De manière symbolique, les épisodes qui retracent, dans les trois fictions, la découverte du sujet de l'énigme se déroulent dans la pénombre, métaphore de l'obscurité hermé neutique. La rencontre de chasseur d'ivoire, dans Heart of Darkness, s'effectue au moment 
où celui-ci, mourant, participe plus de l'absence que de la présence. Le discours de l'excès attendu par Marlow prend place dans le récit sous la forme paradoxale de la lacune, comme l'a analysé Tzvetan Todorov dans un article au titre suggestif : «Connaissance du vide $»^{56}$. Les dernières paroles de Kurtz, "The horror! The horror! $\aleph^{57}$, résumé de l'expérience, visage terrifiant de la vérité à en croire Marlow, apparaissent bien plutôt, selon les termes de Peter Brook, « comme un langage minimal, langage prêt à retomber dans la sauvagerie, prêt à abandonner le langage $»^{58}$. L'initiation attendue par Marlow manifeste dans les derniers moments du récit sa dimension de leurre, aucune révélation précise n'est actualisée dans l'espace du récit. Cette déception finale semble annoncée par la scène de la découverte du chasseur d'ivoire, d'autant plus énigmatique qu'elle demeure muette. «I could not hear a sound $»^{59}$, se rappellera Marlow dont les jumelles découpent un champ optique dans lequel Kurtz apparaît comme un comportement surpris de loin : "I saw the man on the stretcher sit up, lank and with an uplifted hand, above the shoulders of the bearers [...], through my glasses I saw the thin arm extended commingly $"^{60}$. Le sens de ce geste est laissé à son obscurité à l'instar de celui de la femme noire superbe évoqué dans les pages qui suivent ${ }^{61}$. Marlow ne peut surprendre que le signe de la voracité : «I saw him open his mouth wide $»^{62}$. La scène, cette béance de la bouche, invitent alors à se rappeler que le lexique du vide et du manque domine dans l'évocation du chasseur d'ivoire: «M. Kurtz laked restraint [...] there was something wanting in him [...] he was hollow at the core $"^{63}$.

17 A cette absence de révélation vient faire écho le seul mot renvoyé par Grabot, esclave aveugle, attaché à une meule, aux questions de Perken : «- ... Rien... » ${ }^{64}$. Adoptant le point de vue de Claude, la narration souligne alors la dimension de cette réponse : «ce n'était pas un homme qui ne se souvenait pas, ni qui ne voulait pas répondre, c'était un homme qui disait sa vérité $»^{65}$. Le motif de la lacune apparaît aussi dans l'épisode africain de Voyage au bout de la nuit; sans délivrer aucun message à son successeur, Robinson disparaît de la case à la faveur de la nuit, laissant seule la possibilité de procéder au constat : «Il était parti » ${ }^{66}$. Le motif du manque prend ici des inflexions triviales : «Enfin, moi, c'est la caisse que je regrettais surtout dans cette histoire $»^{67}$, rapportera Bardamu. Repris dans l'avant-dernier chapitre du roman, le thème prend des inflexions plus ambiguës tandis que Robinson, après avoir payé de sa vie son refus de tout compromis, se dote de l'aura du héros de la révolte, de celui qui a su aller au bout de la nuit. Alors que les révélations qu'il semble vouloir faire de son lit d'hôpital se transforment en délire d'agonisant, la tonalité associe pathétique et grotesque : «[...] on voyait bien [...] qu'il tenait malgré tout à nous dire encore des choses... La force lui manquait et aussi les moyens. Il pleurait, il étouffait et il riait tout de suite après $»^{68}$. Organisées autour du thème de la lacune, les trois narrations semblent avoir vocation « à approcher un sujet et à l'éviter ${ }^{69}$, conformément à la formule du narrateur du Docteur Faustus.

Les trois romans témoignent d'une fascination à l'égard de cette altérité incarnée par Kurtz avant Grabot, Perken et Robinson mais stratégie narrative et procédés langagiers se conjuguent pour, tout à la fois, approcher ce sujet et l'éviter. A cet égard, Heart of Darkness propose un dispositif narratif complexe qui a pour effet d'ombrer la personnalité de Kurtz et de voiler la violence de son expérience. Le barrage principal établi par le texte tient au redoublement des instances narratives et à la délégation du point de vue à différents personnages - comptable de la station, briquetier, directeur du poste central et son neveu, jeune Russe, épigone du chasseur d'ivoire qui offrent une perception réfractée, surplombée par celle de Marlow et, conjointement, déformée. Un autre barrage opposé 
par le texte consiste dans le jeu de retard temporel. Les temps verbaux associés à Kurtz sont le passé, puisque tout le récit est le fruit d'une anamnèse, et le futur, le conteur procédant à un usage répété de la prolepse, bien plus que le temps de l'actualisation : « des obstacles proprement narratifs s'ajoutent à ceux que dresse la jungle: au lieu de poursuivre son récit de connaissance progressive de Kurtz, Marlow s'interrompt brusquement et dresse un portrait rétrospectif de son héros, comme si Kurtz ne pouvait être présent que dans les temps de l'absence $»^{70}$, constate Tzvetan Todorov. Dans La Voie royale, l'autre est constitué en énigme par le jeu de la délégation du point de vue. A cet égard, l'ouverture du roman est exemplaire, qui montre Claude étudiant le visage de Perken. Le système peut devenir encore plus sophistiqué lorsque Claude observe les réactions de Perken regardant Grabot : "Claude devinait la crispation de ses doigts qui cherchaient à s'accrocher, la stupeur d'un homme qui chavirait » ${ }^{71}$. Une réversibilité s'instaure dans l'espace du roman de Malraux; si Claude est d'abord le point focal, un brusque changement de perspective se produit dans l'épisode de la découverte des blocs sculptés tandis que la narration adopte le point de vue de Perken : « il avait espéré que [la pierre] céderait au premier coup [....]. Une seconde, il vit ses défilés sans mitrailleuse ravagés, bouleversés comme par le passage des éléphants sauvages $»^{72}$. Alors éphémère, ce glissement apparaît à nouveau dans la troisième partie, au moment où Perken choisit délibérément de s'avancer vers les Moïs qui encerclent les trois Occidentaux. Amorcé par une phrase brève, "Perken n'entendait plus " $^{73}$, qui signale que le personnage s'est tourné vers une voix intérieure, le mouvement se poursuit au style indirect libre. Dira-ton que cette réversibilité invite à distinguer le roman de Malraux de ceux de Céline et de Conrad? En fait, lorsque Perken devient intériorité, Claude est à son tour constitué en extériorité. C'est ainsi que se répondent, de manière exemplaire, incipit et excipit du roman: «Claude [...] regardait opiniâtrement le visage de cet homme $»^{74}$, «Perken regardait ce témoin, étranger comme un être d'un autre monde $»^{75}$.

L'opacité d'autrui est encore signifiée, dans le roman de Malraux, à grand renfort d'interrogations. Claude est d'abord le point focal du récit, le narrateur livre les répercussions sur la conscience du jeune homme des propos de Perken, celui-ci demeurant opaque. Dès lors se succèdent, surtout dans les premiers temps du récit, interrogations directes et indirectes: " 'lesquelles?' se demandait Claude », "'sadique?' se demandait maintenant Claude $»^{76}$, « cette conversation était-elle orientée ? », « 'Veut-il dormir, se demanda Claude, ou rompre cette conversation?" »", «Claude s'était d'abord demandé pourquoi Perken avait accepté sa présence ${ }^{78}$, «Allait-il enfin répondre ? ${ }^{79}$, «Claude se demandait s'il réfléchissait », « 'Que connais-je de cet homme ?' pensait-il une fois de plus $\aleph^{80}$. Dans la troisième partie du roman, Grabot devient un objet d'interrogation tandis que la même hypothèse hante les deux protagonistes, d'abord mise au compte de Perken : « ou alors il aurait été tellement pris par la sauvagerie... [...] ... qu'il serait tout à fait transformé $»^{81}$ avant d'être portée à celui de Claude : "la phrase que Perken avait dite dans la clairière retomba sur Claude [...] : 'A moins qu'il n'ait été pris par la sauvagerie...' $»^{82}$. Adoptant la troisième personne pour parler de lui-même, le créateur de Bardamu décrit le dispositif de son roman de manière à mettre au premier plan la question de parler d'autrui: "Céline fait délirer Bardamu qui dit ce qu'il sait de Robinson $"^{83}$. De manière moins ostentatoire que dans $L a$ Voie royale, les modalités narratives, dans Voyage au bout de la nuit, ont encore pour effet de conférer à l'autre le statut d'extériorité. En dépit du caractère rétrospectif de la narration, nul savoir en surplomb ne vient contredire la délégation au point de vue d'un héros dont les interprétations demeurent aléatoires : «je lui trouvai, en l'observant [Robinson], par la 
suite, une figure décidément aventureuse, une figure à angles très tracés et même une de ces têtes de révolte qui entrent trop à vif dans l'existence au lieu de rouler dessus $»^{84}$, tel est l'essai de physiognomonie auquel se livre Bardamu dans l'épisode africain. Cependant, le choix de l'expression «ce ténébreux ${ }^{85}$ pour désigner Robinson dans ce moment du texte sonne peut-être de manière ironique tout en invitant à privilégier l'acception de « difficile à comprendre ». Plus loin, un propos de Bardamu sanctionnera le caractère aporétique de la tentative de connaître autrui :

Pour faire psychologue, nous [la maîtresse de Robinson et Bardamu] essayâmes d'analyser un peu le caractère de Robinson... et je me suis lancé dans une définition de son caractère à Robinson comme si je le connaissais, moi son caractère, mais je me suis aperçu tout de suite que je ne connaissais guère Robinson sauf pour quelques grossières évidences de son tempérament ${ }^{86}$.

Mis en relief, dans les trois fictions, par les modalités de la narration, le thème de l'obscurité de l'autre prend des résonances ontologiques et s'infléchit vers celui d'un solipsisme irréductible des consciences.

Interrompant le fil de son récit, l'ancien capitaine de vapeur procède, dans Heart of Darkness, au constat désabusé d'une solitude ontologique: "We live, as we dream - alone... $»^{87}$. Marlow n'est cependant pas le seul narrateur d'un roman dont l'incipit et l' excipit sont pris en charge par un narrateur anonyme et homodiégétique. Celui-ci reprend donc la conduite du récit à la fin du texte et, passant sous silence les commentaires des autres auditeurs, précise : "[Marlow] sat apart, indistinct and silent $»^{88}$. La précision spatiale fait la part belle au symbolique, et semble sanctionner l'échec à se faire entendre de ses destinataires. Sous son apparente banalité, elle file le propos tragique tenu par Marlow et inscrit in fine le motif du solipsisme des êtres ${ }^{89}$. Celui-ci est repris et se dote d'une présence insistante dans $L a$ Voie royale sous le nom de la "séparation». La présentation inaugurale orchestre un thème également liminaire dans $L a$ condition humaine, qui décrit «les inflexions de la voix de Perken, par sa façon de dire 'ils' en parlant des passagers - et peut-être des hommes - comme s'il eût été séparé d'eux ${ }^{90}$. L'adjectif revient à propos de Grabot présenté en ces termes par Perken: "À cause du courage, il est beaucoup plus séparé du monde que vous ou moi $»^{91}$. Cependant, il s'agit d'évoquer non pas une solitude qui se confondrait avec une simple marginalité sociale mais l'expérience de la conscience séparée ou de la "déréliction originelle», pour reprendre une expression d'Heidegger. Les derniers mots du roman : «Perken regardait ce témoin, étranger comme un être d'un autre monde $»^{92}$ apportent un démenti catégorique et définitif à l'illusion de Claude qui éprouve dans le même temps «cette fraternité désespérée qui le jetait hors de lui-même ${ }^{93}$. Ce thème du malentendu qui consacre la solitude des individus est orchestré à deux reprises avant d'offrir son point d'orgue au roman de Malraux. La première occurrence s'inscrit dans un contexte où la narration adopte le point de vue de Perken. En vertu d'un retournement remarquable, le signe de l'empathie conduit à la prise de conscience de la séparation :

Il y avait en ce regard [de Claude] une complicité intense où se heurtait la poignante fraternité du courage et la compassion, l'union animale des êtres devant la chair condamnée ; Perken, bien qu'il s'attachât à lui plus qu'il ne s'était attaché à aucun être, sentait sa mort comme si elle lui fût venue de lui ${ }^{94}$.

Conformément au principe de symétrie qui gouverne l'organisation de La Voie royale, le personnage de Claude deviendra le sujet d'une telle expérience : 
Ils se regardaient, soumis à ce lien silencieux qui plusieurs fois déjà les avait unis.

[...] Et soudain Claude découvrit combien Perken était plus vieux que lui [...]:

irrémédiablement différent, d'une autre race ${ }^{95}$.

thème de la fraternité pour mieux conclure à son impossibilité. Que la fraternité n'est qu'un leurre, tel est encore l'enseignement de l'épisode africain dans Voyage au bout de la nuit. Le motif apparaît ainsi sous une forme dégradée et triviale; dans la nuit, après avoir redouté d'être assassiné par l'individu dont il rallié la factory, Bardamu est l'objet d'une étrange révélation quant à l'identité de celui-ci, laquelle se conclut par : «il [Robinson] pouvait compter sur mon silence et ma complicité $»^{96}$. Pour être associé, en contexte, aux domaines de la faute et du délit, le terme de « complicité » est dévalué, et bien peu noble par rapport à la « fraternité » évoquée à plusieurs reprises dans La Voie royale. Il n'en reste pas moins qu'il a partie liée par son étymologie avec « une union étroite » et qu'il signifie, par extension, «une entente profonde ». Or, là encore, le désir de connivence prend l'allure du malentendu, Robinson ayant disparu à la faveur de la nuit. A l'instar de Heart of Darkness et de La Voie royale, Voyage au bout de la nuit se clôt sur le thème du solipsisme. Comme dans le roman de Malraux, la mort propose sa consécration au motif. C'est ainsi que Bardamu décrit son impossibilité à partager la souffrance de Robinson agonisant: «Et je restais, devant Léon, pour compatir et jamais j'avais été aussi gêné. J’y arrivais pas... Il ne me trouvait pas. Il en bavait $»^{97}$.

La clôture du chapitre, "Dans la chambre, ça faisait comme un étranger à présent Robinson, qui viendrait d'un pays atroce et qu'on n'oserait plus lui parler ${ }^{98}$, semble proposer un écho fidèle à la formule finale de La Voie royale : « Perken regardait ce témoin, étranger comme un être d'un autre monde $"^{99}$. Quels que soient les légers décalages, abandon du point de vue du moribond au profit de celui de l'observateur, déplacement de l'outil de la comparaison, amplification de la métaphore qui va dans le sens d'un pathé tique accru, la phrase de Céline reprend très précisément la comparaison qui instaure une équivalence entre solipsisme et extranéité géographique radicale, "pays atroce» ou " autre monde », expression dépourvue ici de toute résonance religieuse.

Si la formule qui fournit la chute de l'avant-dernier chapitre de Voyage au bout de la nuit sonne comme une récriture de l'excipit de La Voie royale, la fin du dernier chapitre du roman semble, quant à elle, se souvenir du paragraphe final de Heart of Darkness. Consacré au récit des différentes formalités administratives liées au meurtre de Robinson, le dernier chapitre du roman de Céline est aussi scandé par des évocations des rives de la Seine, rappelant que "là-bas tout au loin, c'était la mer $»^{100}$ et préparant les dernières lignes du texte :

De loin, le remorqueur a sifflé ; son appel a passé le pont, encore une arche, une autre, l'écluse, un autre pont, loin, plus loin... Il appelait à lui toutes les péniches du fleuve toutes, et la ville entière, et le ciel et la campagne et nous, tout qu'il emmenait, la Seine aussi, tout qu'on n'en parle plus ${ }^{101}$.

C'est déjà sur l'évocation d'un univers fluvial que s'achevait Heart of Darkness : « The offing was barred by a black bank of clouds, and the tranquil waterway leading to the uttermost ends of the earth flowed sombre under an overcast sky - seemed to lead into the heart of an immense darkness $»^{102}$. L'excipit de Voyage au bout de la nuit, après celui de Heart of Darkness, propose la description d'un univers étranger à lui-même d'où toute présence humaine se trouve bannie, monde du silence ou des ténèbres. 


\section{NOTES}

1. Et plus particulièrement l'épisode africain dans les chapitres 10 à 14 du roman.

2. Le cadre de cette étude est bien celui de la question de la récriture et non de la simple intertextualité. La seconde notion déplace la perspective du point de vue de la production à celui de la réception. Pour une confrontation de ces deux notions, on peut se reporter au chapitre liminaire de la thèse d'Anne-Claire Gignoux : « La récriture : forme, enjeux, valeurs autour du nouveau roman ", thèse de doctorat, Université Paris 4, 1996, pp. 21-52. 3. Dans le deuxième tome de ses Mémoires, Nos vingt ans, Clara Malraux évoque en ces termes le moment qui précède le départ pour l'Indochine : « On a toujours trop lu. Ma rêverie se souvenait - ou pressentait-elle? - de Coeur des ténèbres : nous aussi nous allions remonter le fleuve, retourner vers nous-mêmes » (Clara Malraux, Nos vingt ans, Grasset, 1992, Les cahiers rouges, p. 90).

4. Milton Hindus, L.-F. Céline tel que je l'ai vu, Paris, L'Herne, lettre du 12 juin 1947, p. 147.

5. Le fait n'est pas établi. L'inverse est mieux connu ; au sein du comité de lecture de Gallimard, le manuscrit de Céline a été soutenu par Berl et Malraux. Dans un entretien accordé par celui-ci à Frédéric Grover en 1973 et consacré intégralement à LouisFerdinand Céline, il n'est nullement question des rapports que La Voie royale et Voyage au bout de la nuit peuvent entretenir, pas plus que de leur dette commune à l'égard de Heart of Darkness (Frédéric J. Grover, Six entretiens avec André Malraux sur des écrivains de son temps, Gallimard, 1978, Idées, « Louis-Ferdinand Céline »). On peut seulement remarquer que Malraux fait un sort particulier au premier roman de Céline, le seul, selon lui, dans lequel l'écrivain « avait à dire des choses importantes » (ibid., p. 86) et se montre singulièrement sensible à l'épisode africain : « Son expérience coloniale n'était pas rien non plus » (ibid., p. 87). Comme Frédéric Grover l'invite à le faire, Malraux préfère s'engager sur la voie d'une comparaison de Voyage au bout de la nuit et de La condition humaine, exercice qui conduit nécessairement au constat d'une opposition entre ces deux romans publiés à un an d'intervalle. Malraux souligne ainsi « l'absence de toute collectivité dans le Voyage. La notion collective domine La condition humaine " (ibid., p. 97). Or, La Voie royale, roman de l'aventurier et non du militant, a bien en partage avec Voyage au bout de la nuit de mettre au premier plan l'individualisme au mépris de tout idéal collectif.

6. Louis-Ferdinand Céline, Bagatelles pour un massacre, Paris, Denoël, 1937, p. 215.

7. Les analogies entre Heart of Darkness et Voyage au bout de la nuit, ces deux romans aux titres étonnamment voisins, ont déjà donné lieu à différentes remarques. Dans un article publié dans L'Etudiant socialiste en janvier 1933, Claude Lévi-Strauss, le premier, invitait à établir un rapprochement entre deux récits dont les narrateurs s'enfoncent au cœur de l'Afrique pour y retrouver un autre Européen : «Que de noms surgissent quand on lit cette évocation coloniale [les tribulations africaines de Bardamu] ! On pense à Conrad, mais à un Conrad dont les brumes de poésie et de mystère se seraient coagulées et solidifiées en arêtes coupantes; où les aventuriers sont plus simplement des exploiteurs et des escrocs, les indigènes secrets, des imbéciles, le cerveau des Européens [...], une masse moisissante d'alcool, de vérole et de fièvre » (cité dans 70 critiques de Voyage au bout de la nuit, IMEC, 1993, p. 120). Plus récemment, Marie-Christine Bellosta invite à reconnaitre, dans le propos tenu dans Voyage au bout de la nuit sur la colonisation, une 
double référence, à Heart of Darkness et à Robinson Crusoe (Marie-Christine Bellosta, Céline ou l'art de la contradiction, Paris, PUF, 1990, « Ce que c'est que la colonisation », pp. 55-58) et elle souligne plus particulièrement cette affinité entre Céline et Conrad : « la description est orientée par le même fantasme : la sensation de la fragilité de l'état de civilisation, la fascination et la peur du retour à la barbarie » (ibid., 56). Dans Céline écrivain, Anne Henry invoque le souvenir de Heart of Darkness dans le traitement du personnage de Robinson, ce double de Bardamu, avant d'émettre une réserve assortie de l'hypothèse que Le compagnon secret constitue ici le véritable hypotexte (Anne Henry, Céline écrivain, Paris, l'Harmattan, 1994, p. 156). Sous le titre, « Chacun sa place. L'anticolonialisme dans Heart of Darkness (1899) et Voyage au bout de la nuit (1932) », Luc Rasson consacre un article aux ambiguités des discours tenus sur l'autre africain. S'il invite à remarquer l'identité de parcours interprétatifs, « un trajet narratif superposable, une expérience analogue de dépaysement herméneutique, un scepticisme partagé sur le projet colonialiste et enfin une même schizophrénie discursive » (Luc Rasson, "Chacun sa place. L'anticolonialisme dans Heart of Darkness et Voyage au bout de la nuit », L'Exotisme, Cahiers CRLH-CIRAOI ${ }^{\circ} 5$, pp. 267-280, p. 273.), il se refuse à établir que le roman de Céline constitue une récriture de celui de Conrad et procède aux réserves suivantes : « Est-ce à dire que Céline, en écrivant son roman, s'est souvenu du récit de Conrad ? La chose n'est pas établie. On ne trouve pas de références à l'œuvre de Conrad, ni dans la correspondance africaine de Louis Destouches [...] ni dans celle contemporaine de la rédaction de Voyage. [...] Les analogies repérées entre les deux textes ne doivent pas pointer vers une influence directe de Conrad. Il faut faire la part du discours européen sur l'Afrique » (ibid., p. 273).

Sous les titres « Malraux et Conrad : un certain roman d'aventure » (in Le livre dans la vie et l'œuvre d'André Malraux, Klincksieck, 1988) et "Malraux et Conrad " (in André Malraux. Unité de l'œuvre, unité de l'homme, La documentation française, 1989), Christiane Moatti propose deux articles presque identiques dans lesquels elle traite très rapidement la question des rapports de Heart of Darkness et de La Voie royale. Dans les mêmes termes, elle attire l'attention sur la similitude des décors : "Le désert végétal de Cœur des ténèbres est très proche de la forêt pourrissante de La Voie royale. C'est l'embuscade perpétuellement tendue par une nature hostile» ("Malraux et Conrad : un certain roman d'aventures ", op. cit., p. 105, "Malraux et Conrad», op. cit., p. 133), et sur l'identité de structures en forme de recherche de «deux individus hors du commun, tous deux transformés par les atteintes sournoises de la sauvagerie, quoique d'une manière différente » ( ibid., p. 106 et 133). Dans la notice de la nouvelle édition critique des romans de Malraux dans la Bibliothèque de la Pléiade, Walter Langlois dégage les mêmes analogies et invite aussi à repérer une semblable critique du colonialisme et « l'utilisation de l'aventure romanesque pour révéler la condition de l'homme» (André Malraux, Euvres complètes, Gallimard, Pléiade, 1989, pp. 1133-1134).

8. Joseph Conrad, Heart of Darkness, Livre de poche $n^{\circ} 8703$, édition bilingue, pp. 40-42 : « Or quand j'étais gosse, j'avais la passion des cartes [...] à l'époque, il y avait un grand nombre d'espaces vides sur la terre [...]. En vérité, depuis mon enfance, ce n'était plus un espace vide. Il avait eu le temps de se remplir de rivières, de lacs, de noms. Ce n'était plus un espace vide de mystère merveilleux, une étendue vierge faite pour inspirer des rêves grandioses à un jeune garçon ».

9. André Malraux, La condition humaine, Livre de poche, p. 19.

10. André Malraux, La Voie royale in Euvres, Gallimard, Bibliothèque de la Pléiade, p. 180.

11. Ibid., p. 178. 
12. Louis-Ferdinand Céline, Voyage au bout de la nuit, Gallimard, Folio $n^{\circ} 28$, p. 168. La pagination, dans la collection Folio, varie selon la date d'impression des exemplaires. Celle que je propose est valable pour les exemplaires imprimés en 1990.

13. Ibid., p. 148.

14. Ibid., p. 188.

15. Ibid., p. 189.

16. Ibid., p. 211.

17. Ibidem.

18. Présent depuis le titre, le réseau sémantique de l'ombre envahit littéralement l'espace du roman de Louis-Ferdinand Céline après celui de Conrad.

19. La Voie royale, éd. cit., p. 186.

20. Voyage au bout de la nuit, éd. cit., p. 217.

21. Heart of Darkness, éd. cit., p. 152 : « pour moi, il [le vapeur] progressait vers Kurtz exclusivement $»$.

22. La voie royale, éd. cit., p. 249.

23. Michel Serres, Jouvences sur Jules Verne, Editions de Minuit, 1974, préface.

24. La Voie royale, éd. cit., p. 197.

25. Voyage au bout de la nuit, éd. cit., p. 228.

26. Heart of Darkness, éd. cit., p. 32 : «Une fascination, aussi, commence à le travailler ».

27. Ibid., p. 54 : « le mental change sur place».

28. Ibid., p. 90 : « je me souvins du vieux médecin, - il serait intéressant pour la science d'observer les changements qui affectent l'esprit des individus, sur place ».

29. La Voie royale, éd. cit., p. 181.

30. Gaëtan Picon, Malraux, Paris, Seuil, 1953, p. 29.

31. Voyage au bout de la nuit, éd. cit., p. 390.

32. Lord Jim, Edinburgh, London, William Blackwood and Sons, 1900.

33. La Voie royale, éd. cit., p. 176.

34. Ibid., p. 177.

35. Ibid., p. 179.

36. Ibid., p. 183.

37. Ibid., p. 187.

38. Ibid., p. 215.

39. Ibid., p. 267.

40. Voyage au bout de la nuit, éd. cit., p. 216.

41. Ibid., p. 15.

42. Ibid., p. 211.

43. Ibid., p. 220.

44. Ibid., p. 227.

45. La Voie royale, éd. cit., p. 175 n. m.

46. Ibid., p. 181.

47. Ibid., page 176. La voix de la rumeur est tout aussi prolixe pour construire des mythes autour de Grabot et de Mayrena, autres figures d'aventuriers du roman.

48. « [the Company's chief accountant] said he was a first-class agent ; and [...] he added slowly, laying down his pen, 'he is a very remarkable person' " (Heart of Darkness, éd. cit., p. 84), « [the manager of the Central Station] began again, assuring me M. Kurtz was the best agent he had, an exceptionnal man, of the greatest importance to the Company » ( ibid., p. 100), " 'He is a prodigy', [the brickmaker of the Central Station] said at last. 'He is an emissary of pity, and science, and progress, and devil knows what else » (ibid., p. 110), 
« Hadn't I been told in all the tones of jealousy and admiration that he had collected, bartered, swindled, or stolen more ivory than all the other agents together? » (ibid,, p. 200).

49. Voyage au bout de la nuit, éd. cit., p. 169.

50. Ibid., p. 171.

51. Ibid., p. 172.

52. Joseph Conrad, « An Outpost of progress » in Tales of Unrest, London, T. Fisher Unwin, 1898.

53. Voyage au bout de la nuit, éd. cit., p. 177.

54. Ibid., p. 188.

55. Un des instruments les plus évidents de la satire du colonialisme réside, dans Voyage au bout de la nuit après Heart of Darkness, dans le recours à l'ironie et à l'antiphrase. Luc Rasson a relevé ce trait que les deux romans ont en partage et il en fait le signe du décalage entre le personnage européen et « sa communauté d'origine » ("Chacun sa place. L'anticolonialisme dans Heart of Darkness et Voyage au bout de la nuit », op. cit., p. 271). La critique du colonialisme est beaucoup plus discrète dans La Voie royale.

56. Tzvetan Todorov, «Connaissance du vide », Nouvelle revue de psychanalyse, printemps 1975, pp.145-154.

57. Ibid., p. 292.

58. Peter Brook, « Un rapport illisible: Au cœur des ténèbres », Poétique, n 44, nov. 1980, p. 479.

59. Heart of Darkness, éd. cit., p. 251 : « Je n'entendais rien ».

60. Ibidem. : « Je vis l'homme sur la civière se redresser, décharné et avec un bras levé, au dessus des épaules des porteurs [...], dans les jumelles, je vis le bras maigre tendu avec autorité ».

61. "Suddenly [the wild and gorgeous woman] opened her bared arms and threw them up rigid above her head, as thought in an uncontrolled desire to touch the sky » (ibid., p. 258).

62. Ibid., p. 252 : « je le vis ouvrir grand la bouche».

63. Ibid., p. $244:$ : M. Kurtz manquait de retenue [...] quelque chose lui faisait défaut [...] il était creux à l'intérieur ».

64. La Voie royale, éd. cit., p. 261.

65. Ibidem. On peut alors reprendre la comparaison établie par Walter Langlois entre Heart of Darkness et $L a$ Voie royale : « Il est [...] évident qu'au héros de Conrad qui agonise et à ses confidences à l'ami qui l'accompagne répondent, dans la dernière partie du texte de Malraux, l'agonie de Perken et ses confidences à Claude Vannec » (Notice à La Voie royale, Pléiade, 1989, op. cit., p. 1134) pour en signaler le caractère hâtif. Désigner Marlow comme « l'ami » de Kurtz ne rend pas compte de la teneur de relations qui tiennent de l'affrontement et d'une complicité dont le caractère paradoxal est ainsi formulé : «je suis resté fidèle, dira Marlow aux passagers de la Nellie, au cauchemar de mon choix ». De plus, le motif qui revient d'un roman à l'autre est celui de la remarquable absence non seulement de " confidences », terme qui semble impropre car trop lié au domaine de l'intimité, mais même de toute révélation ou message.

66. Voyage au bout de la nuit, éd. cit., p. 221.

67. Ibidem.

68. Ibid., p. 627.

69. Thomas Mann, Le docteur Faustus, Paris, Albin Michel, 1950, p. 117.

70. «Connaissance du vide », op. cit., p. 150. 
71. La Voie royale, éd. cit., p. 259.

72. Ibid., p. 236.

73. Ibid., p. 270.

74. Ibid., p. 175.

75. Ibid., p. 310.

76. Ibid., p. 176.

77. Ibid., p. 178.

78. Ibid., p. 182.

79. Ibid., p. 192.

80. Ibid., p. 196.

81. Ibid., p. 249.

82. Ibid., p. 257.

83. Cahiers Céline $\mathrm{n}^{\circ}$ 1, Paris, Gallimard, 1976, p. 31.

84. Voyage au bout de la nuit, éd. cit., p. 212.

85. Ibid., p. 217. Pas plus qu'en nouveau Robinson Crusoe, Léon Robinson ne saurait apparaître en avatar du héros d'Amadis de Gaule.

86. Voyage au bout de la nuit, éd. cit., p. 499.

87. Heart of Darkness, éd. cit., p. 120 : « nous vivons comme nous rêvons, seuls ».

88. Ibid., p. 326 : « était assis à l'écart, invisible et silencieux ».

89. Anne Henry invite ici à repérer un thème schopenhauerien : « l'impossibilité du regard intérieur " (Céline écrivain, éd. cit., p. 156).

90. La voie royale, éd. cit., p. 180.

91. Ibid., p. 244.

92. Ibid., p. 310.

93. Ibidem.

94. Ibid., p. 288.

95. Ibid., p. 289.

96. Voyage au bout de la nuit, éd. cit., p. 220.

97. Ibid., p. 626. On comprend mal l'interprétation proposée par Anne Henry qui évoque Robinson comme « ce personnage pour lequel il [Bardamu] n'éprouve pas de véritable sympathie car il s'agit de toute autre chose comme il le comprend après la mort de son ami » (Céline écrivain, éd. cit., p. 156). Aucune illumination n'intervient pour Bardamu dans ce moment du récit sinon cette compréhension paradoxale d'un écart incommensurable entre les êtres.

98. Ibid., p. 628.

99. La Voie royale, éd. cit., p. 310.

100. Voyage au bout de la nuit, éd. cit., p. 630.

101. Ibid., p. 636.

102. Ibid., p. 326 : « En direction du large s'élevait une barrière de nuages noirs, et le cours d'eau tranquille menant aux confins de la terre coulait sombrement sous un ciel couvert, comme s'il menait au cœur d'infinies ténèbres ». 


\section{RÉSUMÉS}

Il s'agit de lire La Voie royale publiée par Malraux en 1930 et Voyage au bout de la nuit que Céline fait paraître deux ans plus tard comme deux hypertextes du roman de Conrad, Heart of Darkness (1901). Un tel projet semble pouvoir être justifié par une étude comparée du traitement du thème de l'altérité dans les trois fictions. Les trois récits disent à leur manière que le temps des découvertes et échu et qu'au motif de la découverte de territoires vierges, ce topos de la littérature d'aventure, se substitue celui de l'exploration d'autrui. Dès lors, la traversée de territoires, jungle africaine ou cambodgienne adopte pour les héros des fictions la forme de la télémachie. A l'horizon du déplacement se dessine une possible communion d'expérience et les trois textes soulignent, selon des modalités qui leur sont propres, l'identité des parcours et des destins. Pourtant, dans le même temps, les narrations se montrent tout aussi attachées à signifier l'opacité d'autrui et les scènes de rencontre qui devraient offrir un point d'orgue aux parcours s'organisent autour du motif de la lacune. Dès lors, l'enseignement de ces romans qui empruntent volontiers un tour aphoristique est similaire : la fraternité est dénoncée comme une illusion qui dissimule l'expérience authentique de la "séparation", pour reprendre un des leitmotive orchestrés par La Voie royale.

\section{INDEX}

Mots-clés : Joseph Conrad

Index chronologique : XXe siècle

\section{AUTEUR}

\section{ISABELLE GUILLAUME}

Université de Pau 